\title{
Some spectral properties of uniform hypergraphs
}

\author{
Jiang Zhou* \\ College of Science \\ College of Computer Science and Technology \\ Harbin Engineering University \\ Harbin, PR China \\ zhoujiang04113112@163.com \\ Wenzhe Wang \\ College of Science \\ Harbin Engineering University \\ Harbin, PR China \\ 690564734@qq.com \\ Lizhu Sun \\ School of Science \\ Harbin Institute of Technology \\ Harbin, PR China \\ sunlizhu678876@126.com \\ Changjiang Bu \\ College of Science \\ Harbin Engineering University \\ Harbin, PR China \\ buchangjiang@hrbeu.edu.cn
}

Submitted: Jun 5, 2014; Accepted: Oct 22, 2014; Published: Oct 30, 2014

Mathematics Subject Classifications: 05C65, 15A69, 15A18

\begin{abstract}
For a $k$-uniform hypergraph $H$, we obtain some trace formulas for the Laplacian tensor of $H$, which imply that $\sum_{i=1}^{n} d_{i}^{s}(s=1, \ldots, k)$ is determined by the Laplacian spectrum of $H$, where $d_{1}, \ldots, d_{n}$ is the degree sequence of $H$. Using trace formulas for the Laplacian tensor, we obtain expressions for some coefficients of the Laplacian polynomial of a regular hypergraph. We give some spectral characterizations of oddbipartite hypergraphs, and give a partial answer to a question posed by Shao et al (2014). We also give some spectral properties of power hypergraphs, and show that a conjecture posed by $\mathrm{Hu}$ et al (2013) holds under certain conditons.
\end{abstract}

Keywords: Hypergraph eigenvalue; Adjacency tensor; Laplacian tensor; Signless Laplacian tensor; Power hypergraph

\section{Introduction}

Recently, the research on spectral theory of hypergraphs has attracted extensive attention $[1,5-8,11,13,14,16-18]$. We first introduce some necessary concepts and notations. For a

\footnotetext{
*Corresponding author.
} 
positive integer $n$, let $[n]=\{1, \ldots, n\}$. An order $k$ dimension $n$ tensor $\mathcal{A}=\left(a_{i_{1} \cdots i_{k}}\right) \in$ $\mathbb{C}^{n \times \cdots \times n}$ is a multidimensional array with $n^{k}$ entries, where $i_{j} \in[n], j=1, \ldots, k$. We sometimes write $a_{i_{1} \cdots i_{k}}$ as $a_{i_{1} \alpha}$, where $\alpha=i_{2} \cdots i_{k}$. When $k=1, \mathcal{A}$ is a column vector of dimension $n$. When $k=2, \mathcal{A}$ is an $n \times n$ matrix. The unit tensor of order $k \geqslant 2$ and dimension $n$ is a diagonal tensor $\mathcal{I}_{n}=\left(\delta_{i_{1} i_{2} \cdots i_{k}}\right)$ such that $\delta_{i_{1} i_{2} \cdots i_{k}}=1$ if $i_{1}=i_{2}=\cdots=i_{k}$, and $\delta_{i_{1} i_{2} \cdots i_{k}}=0$ otherwise. In [15], Shao defined the following product of tensors, which is a generalization of the matrix multiplication.

Definition 1. [15] Let $\mathcal{A}$ and $\mathcal{B}$ be order $m \geqslant 2$ and order $k \geqslant 1$, dimension $n$ tensors, respectively. The product $\mathcal{A B}$ is the following tensor $\mathcal{C}$ of order $(m-1)(k-1)+1$ and dimension $n$ with entries

$$
c_{i \alpha_{1} \ldots \alpha_{m-1}}=\sum_{i_{2}, \ldots, i_{m} \in[n]} a_{i i_{2} \ldots i_{m}} b_{i_{2} \alpha_{1}} \cdots b_{i_{m} \alpha_{m-1}}\left(i \in[n], \alpha_{1}, \ldots, \alpha_{m-1} \in[n]^{k-1}\right) .
$$

Let $\mathcal{A}$ be an order $k \geqslant 2$ dimension $n$ tensor, and let $x=\left(x_{1}, \ldots, x_{n}\right)^{\top}$. From Definition 1, the product $\mathcal{A} x$ is a vector in $\mathbb{C}^{n}$ whose $i$-th component is (see Example 1.1 in [15])

$$
(\mathcal{A} x)_{i}=\sum_{i_{2}, \ldots, i_{k} \in[n]} a_{i i_{2} \cdots i_{k}} x_{i_{2}} \cdots x_{i_{k}}
$$

The concept of tensor eigenvalues was posed in [9, 12]. If there exists a nonzero vector $x \in \mathbb{C}^{n}$ such that $\mathcal{A} x=\lambda x^{[k-1]}$, then $\lambda$ is called an eigenvalue of $\mathcal{A}, x$ is an eigenvector of $\lambda$, where $x^{[k-1]}=\left(x_{1}^{k-1}, \ldots, x_{n}^{k-1}\right)^{\top}$. The determinant of $\mathcal{A}$, $\operatorname{denoted} \operatorname{by} \operatorname{det}(\mathcal{A})$, is the resultant of the system of polynomials $f_{i}\left(x_{1}, \ldots, x_{n}\right)=(\mathcal{A} x)_{i}(i=1, \ldots, n)$. The characteristic polynomial of $\mathcal{A}$ is defined as $\phi_{\mathcal{A}}(\lambda)=\operatorname{det}\left(\lambda \mathcal{I}_{n}-\mathcal{A}\right)$, where $\mathcal{I}_{n}$ is the unit tensor of order $k$ and dimension $n$. It is known that eigenvalues of $\mathcal{A}$ are exactly roots of $\phi_{\mathcal{A}}(\lambda)[12]$. The multiset of roots of $\phi_{\mathcal{A}}(\lambda)$ (counting multiplicities) is the spectrum of $\mathcal{A}$, denoted by $\sigma(\mathcal{A})$. The maximal modulus of eigenvalues of $\mathcal{A}$ is called the spectral radius of $\mathcal{A}$, denoted by $\rho(\mathcal{A})$. More details on eigenvalues and characteristic polynomials of tensors can be found in $[4,12]$.

A hypergraph $H$ is called $k$-uniform if each edge of $H$ contains exactly $k$ distinct vertices. Let $V(H)$ and $E(H)$ denote the vertex set and the edge set of $H$, respectively. In [13], Qi defined the Laplacian and the signless Laplacian tensor of a uniform hypergraph as follows.

Definition 2. [7, 13] The adjacency tensor of a $k$-uniform hypergraph $H$, denoted by $\mathcal{A}_{H}$, is an order $k$ dimension $|V(H)|$ tensor with entries

$$
a_{i_{1} i_{2} \cdots i_{k}}= \begin{cases}\frac{1}{(k-1) !} & \text { if } i_{1} i_{2} \cdots i_{k} \in E(H), \\ 0 & \text { otherwise. }\end{cases}
$$

Let $\mathcal{D}_{H}$ be an order $k$ dimension $|V(H)|$ diagonal tensor whose diagonal entries are vertex degrees of $H$. The tensors $\mathcal{L}_{H}=\mathcal{D}_{H}-\mathcal{A}_{H}$ and $\mathcal{Q}_{H}=\mathcal{D}_{H}+\mathcal{A}_{H}$ are the Laplacian 
tensor and the signless Laplacian tensor of $H$, respectively. Eigenvalues of $\mathcal{A}_{H}, \mathcal{L}_{H}$ and $\mathcal{Q}_{H}$ are called eigenvalues, Laplacian eigenvalues and signless Laplacian eigenvalues of $H$, respectively. Characteristic polynomials of $\mathcal{L}_{H}$ and $\mathcal{Q}_{H}$ are called Laplacian polynomial and signless Laplacian polynomial of $H$, respectively.

This paper is organized as follows. In Section 2, we give some trace formulas for the Laplacian tensor of a uniform hypergraph, and obtain expressions for some coefficients of the Laplacian polynomial of a regular hypergraph. In Section 3, we give some spectral characterizations of odd-bipartite hypergraphs. In Section 4, we give some spectral properties of power hypergraphs.

\section{Laplacian spectra and degree sequence of hyper- graphs}

Traces of tensors are very useful in the study of spectral theory of tensors. The $d$-th order trace of an order $k \geqslant 2$ dimension $n$ tensor $\mathcal{T}=\left(t_{i_{1} \cdots i_{k}}\right)$ is defined as $[1,4,10]$

$$
\operatorname{Tr}_{d}(\mathcal{T})=(k-1)^{n-1} \sum_{d_{1}+\cdots+d_{n}=d} \prod_{i=1}^{n} \frac{1}{\left(d_{i}(k-1)\right) !}\left(\sum_{y \in[n]^{k-1}} t_{i y} \frac{\partial}{\partial a_{i y}}\right)^{d_{i}} \operatorname{tr}\left(A^{d(k-1)}\right),
$$

where $A=\left(a_{i j}\right)$ is an $n \times n$ auxiliary matrix, and $\frac{\partial}{\partial a_{i y}}=\frac{\partial}{\partial a_{i i_{2}}} \cdots \frac{\partial}{\partial a_{i i_{k}}}$ if $y=i_{2} \cdots i_{k}$. The codegree $d$ coefficient of the characteristic polynomial of $\mathcal{T}$ can be expressed in terms of $\operatorname{Tr}_{1}(\mathcal{T}), \ldots, \operatorname{Tr}_{d}(\mathcal{T})$ (see [4, Theorem 6.3]). It is also known that $\operatorname{Tr}_{t}(\mathcal{T})=\sum_{\lambda \in \sigma(\mathcal{T})} \lambda^{t}$ for any $t \in\left[n(k-1)^{n-1}\right]$ (see [4, Theorem 6.10]). Hence $\operatorname{Tr}_{d}(\mathcal{T})$ is an important invariant in the spectral theory of tensors.

Shao et al [16] give a graph theoretical formula for $\operatorname{Tr}_{d}(\mathcal{T})$. In order to describe this formula, we introduce some notations in [16]. For an integer $d>0$, we define

$$
\mathcal{F}_{d}=\left\{\left(i_{1} \alpha_{1}, \ldots, i_{d} \alpha_{d}\right) \mid 1 \leqslant i_{1} \leqslant \cdots \leqslant i_{d} \leqslant n ; \alpha_{1}, \ldots, \alpha_{d} \in[n]^{k-1}\right\} .
$$

For $F=\left(i_{1} \alpha_{1}, \ldots, i_{d} \alpha_{d}\right) \in \mathcal{F}_{d}$ and an order $k \geqslant 2$ dimension $n$ tensor $\mathcal{T}=\left(t_{i_{1} \cdots i_{k}}\right)$, we write $\pi_{F}(\mathcal{T})=\prod_{j=1}^{d} t_{i_{j} \alpha_{j}}$. Let $p_{i}(F)$ be the total number of times that the index $i$ appears in $F$. If $p_{i}(F)$ is a multiple of $k$ for any $i \in[n]$, then $F$ is called $k$-valent.

Definition 3. [16] Let $F=\left(i_{1} \alpha_{1}, \ldots, i_{d} \alpha_{d}\right) \in \mathcal{F}_{d}$, where $i_{j} \alpha_{j} \in[n]^{k}, j=1, \ldots, d$. Then (1) Let $E(F)=\bigcup_{j=1}^{d} E_{j}(F)$, where $E_{j}(F)$ is the arc multi-set

$$
E_{j}(F)=\left\{\left(i_{j}, v_{1}\right), \ldots,\left(i_{j}, v_{k-1}\right)\right\}
$$

if $\alpha_{j}=v_{1} \cdots v_{k-1}$.

(2) Let $b(F)$ be the product of the factorials of the multiplicities of all the arcs of $E(F)$.

(3) Let $c(F)$ be the product of the factorials of the outdegrees of all the vertices in the arc multi-set $E(F)$.

(4) Let $W(F)$ be the set of all closed walks $W$ with the arc multi-set $E(F)$. 
Shao et al give a graph theoretical formula for $\operatorname{Tr}_{d}(\mathcal{T})$ as follows (see equation (3.5) in $[16])$.

Lemma 4. [16] Let $\mathcal{T}=\left(T_{i_{1} \cdots i_{k}}\right)$ be an order $k \geqslant 2$ dimension $n$ tensor. Then

$$
\operatorname{Tr}_{d}(\mathcal{T})=(k-1)^{n-1} \sum_{F \in \mathcal{F}_{d}^{\prime}} \frac{b(F)}{c(F)} \pi_{F}(\mathcal{T})|W(F)|,
$$

where $\mathcal{F}_{d}^{\prime}=\left\{F \mid F \in \mathcal{F}_{d}, F\right.$ is k-valent $\}$.

For a $k$-uniform hypergraph $H$, Cooper and Dutle [1] proved that $\operatorname{Tr}_{d}\left(\mathcal{A}_{H}\right)=0$ for $d \in[k-1]$. We give some trace formulas for the Laplacian (signless Laplacian) tensor of uniform hypergraphs as follows.

Theorem 5. Let $H$ be a k-uniform hypergraph with degree sequence $d_{1}, \ldots, d_{n}$. Then

$$
\begin{aligned}
\operatorname{Tr}_{t}\left(\mathcal{L}_{H}\right) & =\operatorname{Tr}_{t}\left(\mathcal{Q}_{H}\right)=(k-1)^{n-1} \sum_{i=1}^{n} d_{i}^{t}, t=1, \ldots, k-1, \\
\operatorname{Tr}_{k}\left(\mathcal{L}_{H}\right) & =(-1)^{k} k^{k-1}(k-1)^{n-k}|E(H)|+(k-1)^{n-1} \sum_{i=1}^{n} d_{i}^{k}, \\
\operatorname{Tr}_{k}\left(\mathcal{Q}_{H}\right) & =k^{k-1}(k-1)^{n-k}|E(H)|+(k-1)^{n-1} \sum_{i=1}^{n} d_{i}^{k} .
\end{aligned}
$$

Proof. By Lemma 4, we have

$$
\operatorname{Tr}_{t}\left(\mathcal{L}_{H}\right)=(k-1)^{n-1} \sum_{F \in \mathcal{F}_{t}^{\prime}} \frac{b(F)}{c(F)} \pi_{F}\left(\mathcal{L}_{H}\right)|W(F)|,
$$

where $\mathcal{F}_{t}^{\prime}=\left\{F \mid F \in \mathcal{F}_{t}, F\right.$ is k-valent $\}$. For $F=\left(i_{1} \alpha_{1}, \ldots, i_{t} \alpha_{t}\right) \in \mathcal{F}_{t}$, if $\pi_{F}\left(\mathcal{L}_{H}\right)=$ $\prod_{j=1}^{t}\left(\mathcal{L}_{H}\right)_{i_{j} \alpha_{j}} \neq 0$, then $i_{j} \alpha_{j}=i_{j} i_{j} \cdots i_{j} \in[n]^{k}$ or $i_{j} \alpha_{j} \in E(H)$ for any $1 \leqslant j \leqslant t$.

Let $F \in \mathcal{F}_{t}$ satisfies $\pi_{F}\left(\mathcal{L}_{H}\right) \neq 0$. If $t<k$, then $F$ is k-valent if and only if $F=$ $\left(i_{1} i_{1} \cdots i_{1}, \ldots, i_{t} i_{t} \cdots i_{t}\right)$. In this case, $|W(F)| \neq 0$ if and only if $i_{1}=\cdots=i_{t}$. Let $F_{i}=(i i \cdots i, \ldots, i i \cdots i) \in \mathcal{F}_{t}^{\prime}(t<k)$. From Eq. (1) and Definition 3, we have

$$
\begin{aligned}
\operatorname{Tr}_{t}\left(\mathcal{L}_{H}\right) & =(k-1)^{n-1} \sum_{i=1}^{n} \frac{b\left(F_{i}\right)}{c\left(F_{i}\right)} \pi_{F_{i}}\left(\mathcal{L}_{H}\right)\left|W\left(F_{i}\right)\right| \\
& =(k-1)^{n-1} \sum_{i=1}^{n} \frac{(t(k-1)) !}{(t(k-1)) !} d_{i}^{t}=(k-1)^{n-1} \sum_{i=1}^{n} d_{i}^{t} .
\end{aligned}
$$

Similar with the above procedure, we can also get $\operatorname{Tr}_{t}\left(\mathcal{Q}_{H}\right)=(k-1)^{n-1} \sum_{i=1}^{n} d_{i}^{t}, t=$ $1, \ldots, k-1$.

Let $F \in \mathcal{F}_{k}$ satisfies $\pi_{F}\left(\mathcal{L}_{H}\right) \neq 0$. Then $F$ is k-valent and $|W(F)| \neq 0$ if and only if $F=(i i \cdots i, \ldots, i i \cdots i)$ or $F=\left(i_{1} \alpha_{1}, \ldots, i_{k} \alpha_{k}\right)$, where $i_{1} \alpha_{1}, \ldots, i_{k} \alpha_{k}$ correspond to the 
same edge $i_{1} i_{2} \cdots i_{k} \in E(H)$. Let $F_{i}=(i i \cdots i, \ldots, i i \cdots i) \in \mathcal{F}_{k}^{\prime}$. From Eq. (1) and Definition 3, we have

$$
\begin{aligned}
\operatorname{Tr}_{k}\left(\mathcal{L}_{H}\right) & =(-1)^{k} \operatorname{Tr}_{k}\left(\mathcal{A}_{H}\right)+(k-1)^{n-1} \sum_{i=1}^{n} \frac{b\left(F_{i}\right)}{c\left(F_{i}\right)} \pi_{F_{i}}\left(\mathcal{L}_{H}\right)\left|W\left(F_{i}\right)\right| \\
& =(-1)^{k} \operatorname{Tr}_{k}\left(\mathcal{A}_{H}\right)+(k-1)^{n-1} \sum_{i=1}^{n} \frac{(k(k-1)) !}{(k(k-1)) !} d_{i}^{k} \\
& =(-1)^{k} \operatorname{Tr}_{k}\left(\mathcal{A}_{H}\right)+(k-1)^{n-1} \sum_{i=1}^{n} d_{i}^{k}
\end{aligned}
$$

From the proof of $\left[1\right.$, Theorem 3.15], we have $\operatorname{Tr}_{k}\left(\mathcal{A}_{H}\right)=k^{k-1}(k-1)^{n-k}|E(H)|$. Hence

$$
\operatorname{Tr}_{k}\left(\mathcal{L}_{H}\right)=(-1)^{k} k^{k-1}(k-1)^{n-k}|E(H)|+(k-1)^{n-1} \sum_{i=1}^{n} d_{i}^{k} .
$$

Similar with the above procedure, we can also get

$$
\operatorname{Tr}_{k}\left(\mathcal{Q}_{H}\right)=k^{k-1}(k-1)^{n-k}|E(H)|+(k-1)^{n-1} \sum_{i=1}^{n} d_{i}^{k} .
$$

Remark. Note that traces of a tensor are determined by its spectrum [3, Theorem 6.3]. For a $k$-uniform hypergraph $H$, by Theorem 5 , we know that $\sum_{i=1}^{n} d_{i}^{s}(s=1, \ldots, k)$ is determined by the Laplacian (signless Laplacian) spectrum of $H$, where $d_{1}, \ldots, d_{n}$ is the degree sequence of $H$.

Let $p_{t}(\mathcal{M})$ denote the codegree $t$ coefficient of the characteristic polynomial of a tensor $\mathcal{M}$.

Lemma 6. Let $\mathcal{M}$ be an order $k \geqslant 2$ dimension $n$ tensor. Then

$$
t ! p_{t}(\mathcal{M})=\operatorname{det}\left(\begin{array}{ccccc}
-T r_{t} & T r_{1} & T r_{2} & \cdots & T r_{t-1} \\
-T r_{t-1} & t-1 & T r_{1} & \cdots & T r_{t-2} \\
-T r_{t-2} & 0 & t-2 & \ddots & \vdots \\
\vdots & \vdots & \ddots & \ddots & T r_{1} \\
-T r_{1} & 0 & \cdots & 0 & 1
\end{array}\right)
$$

where $\operatorname{Tr}_{t}=\operatorname{Tr}_{t}(\mathcal{M}), t \in\left[n(k-1)^{n-1}\right]$.

Proof. From [4, Theorem 6.10], we have

$$
\left(\begin{array}{ccccc}
t & T r_{1} & T r_{2} & \cdots & T r_{t-1} \\
0 & t-1 & T r_{1} & \cdots & T r_{t-2} \\
0 & 0 & t-2 & \ddots & \vdots \\
\vdots & \vdots & \ddots & \ddots & T r_{1} \\
0 & 0 & \cdots & 0 & 1
\end{array}\right)\left(\begin{array}{c}
p_{t}(\mathcal{M}) \\
p_{t-1}(\mathcal{M}) \\
\vdots \\
p_{2}(\mathcal{M}) \\
p_{1}(\mathcal{M})
\end{array}\right)=\left(\begin{array}{c}
-T r_{t} \\
-T r_{t-1} \\
\vdots \\
-T r_{2} \\
-T r_{1}
\end{array}\right)
$$


We can obtain the expression of $t ! p_{t}(\mathcal{M})$ from Cramer's rule.

A uniform hypergraph $H$ is called $d$-regular if each vertex of $H$ has degree $d$. The following are some coefficients of the Laplacian (signless Laplacian) polynomial of regular hypergraphs.

Theorem 7. Let $H$ be a d-regular $k$-uniform hypergraph with $n$ vertices. Then

$$
\begin{aligned}
& p_{t}\left(\mathcal{L}_{H}\right)=p_{t}\left(\mathcal{Q}_{H}\right)=(-1)^{t} d^{t}\left(\begin{array}{c}
n(k-1)^{n-1} \\
t
\end{array}\right), t=1, \ldots, k-1, \\
& p_{k}\left(\mathcal{L}_{H}\right)=(-1)^{k+1} k^{k-3}(k-1)^{n-k} n d+(-1)^{k} d^{k}\left(\begin{array}{c}
n(k-1)^{n-1} \\
k
\end{array}\right), \\
& p_{k}\left(\mathcal{Q}_{H}\right)=-k^{k-3}(k-1)^{n-k} n d+(-1)^{k} d^{k}\left(\begin{array}{c}
n(k-1)^{n-1} \\
k
\end{array}\right) .
\end{aligned}
$$

Proof. By Lemma 6, we have

$$
t ! p_{t}\left(\mathcal{L}_{H}\right)=\operatorname{det}\left(\begin{array}{ccccc}
-T r_{t} & T r_{1} & T r_{2} & \cdots & T r_{t-1} \\
-T r_{t-1} & t-1 & T r_{1} & \cdots & T r_{t-2} \\
-T r_{t-2} & 0 & t-2 & \ddots & \vdots \\
\vdots & \vdots & \ddots & \ddots & T r_{1} \\
-T r_{1} & 0 & \cdots & 0 & 1
\end{array}\right)
$$

where $\operatorname{Tr}_{t}=\operatorname{Tr}_{t}\left(\mathcal{L}_{H}\right)$. Since $H$ is $d$-regular, by Theorem 5 , we have $\operatorname{Tr}_{i}=d \operatorname{Tr}_{i-1}=$ $n(k-1)^{n-1} d^{i}, i=2, \ldots, k-1$. If $t<k$, then by Eq. (2), we have

$$
\begin{aligned}
& t ! p_{t}\left(\mathcal{L}_{H}\right)=\operatorname{det}\left(\begin{array}{ccccc}
0 & \operatorname{Tr}_{1} & \operatorname{Tr}_{2} & \cdots & T r_{t-1} \\
0 & t-1 & \operatorname{Tr}_{1} & \cdots & T r_{t-2} \\
\vdots & 0 & t-2 & \ddots & \vdots \\
0 & \vdots & \ddots & \ddots & T r_{1} \\
d-\operatorname{Tr}_{1} & 0 & \cdots & 0 & 1
\end{array}\right) \\
& =\operatorname{det}\left(\begin{array}{ccccc}
0 & \operatorname{Tr}_{1}-(t-1) d & 0 & \cdots & 0 \\
0 & t-1 & \ddots & \ddots & \vdots \\
\vdots & 0 & \ddots & \operatorname{Tr}_{1}-2 d & 0 \\
0 & \vdots & \ddots & 2 & T r_{1} \\
d-\operatorname{Tr}_{1} & 0 & \cdots & 0 & 1
\end{array}\right) \\
& =(-1)^{t} \prod_{i=0}^{t-1}\left(\operatorname{Tr}_{1}-i d\right) \text {. }
\end{aligned}
$$

Since $\operatorname{Tr}_{1}=n(k-1)^{n-1} d$, we have

$$
\begin{aligned}
p_{t}\left(\mathcal{L}_{H}\right) & =(-1)^{t} \frac{\prod_{i=0}^{t-1}\left(n(k-1)^{n-1} d-i d\right)}{t !}=(-1)^{t} d^{t} \frac{\prod_{i=0}^{t-1}\left(n(k-1)^{n-1}-i\right)}{t !} \\
& =(-1)^{t} d^{t}\left(\begin{array}{c}
n(k-1)^{n-1} \\
t
\end{array}\right) .
\end{aligned}
$$


Similar with the above procedure, we can also get

$$
p_{t}\left(\mathcal{Q}_{H}\right)=(-1)^{t} d^{t}\left(\begin{array}{c}
n(k-1)^{n-1} \\
t
\end{array}\right), t=1, \ldots, k-1 .
$$

Since $H$ is $d$-regular, by Theorem 5 , we have $\operatorname{Tr}_{k}=(-1)^{k} k^{k-2}(k-1)^{n-k} n d+d T r_{k-1}$ and $\operatorname{Tr}_{i}=d \operatorname{Tr}_{i-1}=n(k-1)^{n-1} d^{i}, i=2, \ldots, k-1$. From Eq. (2), we have

$$
\begin{aligned}
& k ! p_{k}\left(\mathcal{L}_{H}\right)=\operatorname{det}\left(\begin{array}{ccccc}
(-1)^{k+1} k^{k-2}(k-1)^{n-k} n d & \operatorname{Tr}_{1} & \operatorname{Tr}_{2} & \cdots & \operatorname{Tr}_{k-1} \\
0 & k-1 & \operatorname{Tr}_{1} & \cdots & \operatorname{Tr}_{k-2} \\
\vdots & 0 & k-2 & \ddots & \vdots \\
0 & \vdots & \ddots & \ddots & \operatorname{Tr}_{1} \\
d-\operatorname{Tr}_{1} & 0 & \cdots & 0 & 1
\end{array}\right) \\
& =\operatorname{det}\left(\begin{array}{ccccc}
(-1)^{k+1} k^{k-2}(k-1)^{n-k} n d & \operatorname{Tr}_{1}-(k-1) d & 0 & \ldots & 0 \\
0 & k-1 & \ddots & \ddots & \vdots \\
\vdots & 0 & \ddots & \operatorname{Tr}_{1}-2 d & 0 \\
0 & \vdots & \ddots & 2 & \operatorname{Tr}_{1} \\
d-\operatorname{Tr}_{1} & 0 & \cdots & 0 & 1
\end{array}\right) \\
& =(-1)^{k+1} k^{k-2}(k-1)^{n-k}(k-1) ! n d+(-1)^{k} d^{k} \prod_{i=0}^{k-1}\left(n(k-1)^{n-1}-i\right) \text {. } \\
& p_{k}\left(\mathcal{L}_{H}\right)=(-1)^{k+1} k^{k-3}(k-1)^{n-k} n d+(-1)^{k} d^{k}\left(\begin{array}{c}
n(k-1)^{n-1} \\
k
\end{array}\right) .
\end{aligned}
$$

Similar with the above procedure, we can also get

$$
p_{k}\left(\mathcal{Q}_{H}\right)=-k^{k-3}(k-1)^{n-k} n d+(-1)^{k} d^{k}\left(\begin{array}{c}
n(k-1)^{n-1} \\
k
\end{array}\right) .
$$

\section{Eigenvalues and odd-bipartite hypergraphs}

A $k$-uniform hypergraph $H$ is called odd-bipartite, if there exists a proper subset $V_{1}$ of $V(H)$ such that each edge of $H$ contains exactly odd number of vertices in $V_{1}[6,17]$. Spectral characterizations of odd-bipartite hypergraphs will be investigated in this section. We first give some auxiliary lemmas. The following lemma can be obtained from equation (2.1) in [15].

Lemma 8. Let $\mathcal{A}=\left(a_{i_{1} \cdots i_{k}}\right)$ be an order $k \geqslant 2$ dimension $n$ tensor, and let $P=\left(p_{i j}\right), Q=$ $\left(q_{i j}\right)$ be $n \times n$ matrices. Then

$$
(P \mathcal{A} Q)_{i_{1} \cdots i_{k}}=\sum_{j_{1}, \ldots, j_{k} \in[n]} a_{j_{1} \cdots j_{k}} p_{i_{1} j_{1}} q_{j_{2} i_{2}} \cdots q_{j_{k} i_{k}}
$$


Lemma 9. [6] Let $H$ be a connected k-uniform hypergraph. A nonzero vector $x$ is an eigenvector of $\mathcal{Q}_{H}$ corresponds to the zero eigenvalue if and only if there exist nonzero $\gamma \in \mathbb{C}$ and integers $\alpha_{i}$ such that $x_{i}=\gamma \exp \left(\frac{2 \alpha_{i} \pi}{k} \sqrt{-1}\right)$ for each $i \in V(H)$, and

$$
\sum_{j \in e} \alpha_{j}=\sigma_{e} k+\frac{k}{2}
$$

for some integer $\sigma_{e}$ associated with each $e \in E(H)$.

Weakly irreducible tensors are defined in [3]. It is known that a $k$-uniform hypergraph $H$ is connected if and only if $\mathcal{A}_{H}$ is weakly irreducible [11].

Lemma 10. $[17,19]$ Let $\mathcal{A}$ be an order $k$ dimension $n$ nonnegative weakly irreducible tensor. If $\rho(\mathcal{A}) \exp (\theta \sqrt{-1})$ is an eigenvalue of $\mathcal{A}$, then there exists a diagonal matrix $U$ with unit diagonal entries such that

$$
\mathcal{A}=\exp (-\theta \sqrt{-1}) U^{-(k-1)} \mathcal{A} U
$$

For a tensor $\mathcal{T}$, let $H \sigma(\mathcal{T})=\{\lambda \mid \lambda \in \sigma(\mathcal{T}), \lambda$ has a real eigenvector $\}$. For a connected $k$-uniform hypergraph $G$, Shao et al [17] proved that

$$
H \sigma\left(\mathcal{L}_{G}\right)=H \sigma\left(\mathcal{Q}_{G}\right) \Longrightarrow \sigma\left(\mathcal{L}_{G}\right)=\sigma\left(\mathcal{Q}_{G}\right) .
$$

Shao et al wish to know whether the reverse implication is true. We show that the reverse is true when $k$ is not divisible by 4 .

Theorem 11. Let $G$ be a connected k-uniform hypergraph, and $k$ is not divisible by 4 . Then the following are equivalent:

(1) $k$ is even and $H$ is odd-bipartite.

(2) $H \sigma\left(\mathcal{L}_{G}\right)=H \sigma\left(\mathcal{Q}_{G}\right)$.

(3) $\sigma\left(\mathcal{L}_{G}\right)=\sigma\left(\mathcal{Q}_{G}\right)$.

(4) 0 is a signless Laplacian eigenvalue of $G$.

Proof. From [17, Theorem 2.2], we have $(1) \Rightarrow(2) \Rightarrow(3)$. Since 0 is always an eigenvalue of $\mathcal{L}_{G}$ (see [13]), we have $(3) \Rightarrow(4)$. Next we prove that $(4) \Rightarrow(1)$.

If 0 is an eigenvalue of $\mathcal{Q}_{G}$, then by Lemma 9 , there exists a vertex labeling $f: V(G) \rightarrow$ $[k]$ such that

$$
\sum_{i \in e} f(i) \equiv \frac{k}{2}(\bmod k)
$$

for each $e \in E(G)$. Hence $k$ is even. Since $k$ is not divisible by 4 , we know that $\frac{k}{2}$ is odd. So $\sum_{i \in e} f(i)$ is odd for each $e \in E(G)$. Let $V_{1}=\{u \mid u \in V(G), f(u)$ is odd $\}$. For any $e \in E(G)$, since $\sum_{i \in e} f(i)$ is odd, $e$ contains exactly odd number of vertices in $V_{1}$. Hence $G$ is odd-bipartite. 
When $k=2$, Theorem 11 becomes a classic result in spectral graph theory, i.e., a connected graph $G$ is bipartite if and only if 0 is a signless Laplacian eigenvalue of $G$. It is also well known that a connected graph $G$ is bipartite if and only if $-\rho\left(\mathcal{A}_{G}\right)$ is an eigenvalue of $G$. We generalize this result as follows.

Theorem 12. Let $H$ be a connected $k$-uniform hypergraph, and $k$ is not divisible by 4 . Then the following are equivalent:

(1) $k$ is even and $H$ is odd-bipartite.

(2) $-\rho\left(\mathcal{A}_{H}\right)$ is an eigenvalue of $H$.

Proof. From [17, Theorem 2.3], we have (1) $\Rightarrow(2)$. If (2) holds, then by Lemma 10, there exists a diagonal matrix $U$ with unit diagonal entries such that $\mathcal{A}_{H}=-U^{-(k-1)} \mathcal{A}_{H} U$. By Lemma 8, we have

$$
a_{i_{1} i_{2} \cdots i_{k}}=-a_{i_{1} i_{2} \cdots i_{k}} u_{i_{1}}^{-(k-1)} u_{i_{2}} \cdots u_{i_{k}},
$$

where $u_{i_{j}}$ is the diagonal entry of $U$ corresponds to vertex $i_{j}(j=1, \ldots, k)$. For any edge $e=i_{1} i_{2} \cdots i_{k} \in E(H)$, we get

$$
u_{i_{1}}^{-(k-1)} u_{i_{2}} \cdots u_{i_{k}}=-1, u_{i_{1}} u_{i_{2}} \cdots u_{i_{k}}=-u_{i_{1}}^{k} .
$$

Similarly, we have $u_{i_{1}} u_{i_{2}} \cdots u_{i_{k}}=-u_{i_{1}}^{k}=\cdots=-u_{i_{k}}^{k}$. Since $u_{i_{1}}, \ldots, u_{i_{k}}$ are unit complex number, there exist $\theta$ and integers $\alpha_{i_{1}}, \ldots, \alpha_{i_{k}}$ such that $u_{i_{j}}=\exp \left(\frac{2 \pi \alpha_{i_{j}}+\theta}{k} \sqrt{-1}\right), j=$ $1, \ldots, k$. Then

$$
\begin{aligned}
u_{i_{1}} u_{i_{2}} \cdots u_{i_{k}}=\exp \left(\frac{k \theta+2 \pi \sum_{j=1}^{k} \alpha_{i_{j}}}{k} \sqrt{-1}\right) & =-u_{i_{1}}^{k}=-\exp (\theta \sqrt{-1}), \\
\exp \left(\frac{2 \pi \sum_{j=1}^{k} \alpha_{i_{j}}}{k} \sqrt{-1}\right) & =-1
\end{aligned}
$$

Hence $\sum_{j=1}^{k} \alpha_{i_{j}} \equiv \frac{k}{2}(\bmod k), k$ is even. Since $k$ is not divisible by $4, \sum_{j=1}^{k} \alpha_{i_{j}}$ is odd for any edge $e=i_{1} i_{2} \cdots i_{k} \in E(H)$. Let $V_{1}=\left\{u \mid u \in V(H), \alpha_{u}\right.$ is odd $\}$. For any $e=i_{1} i_{2} \cdots i_{k} \in E(H)$, since $\sum_{j=1}^{k} \alpha_{i_{j}}$ is odd, $e$ contains exactly odd number of vertices in $V_{1}$. Hence $H$ is odd-bipartite.

Let $H$ be a connected $k$-uniform hypergraph. If 0 is an eigenvalue of $\mathcal{Q}_{H}$, then by the proof of Theorem 11, we know that there exists a vertex labeling $f: V(H) \rightarrow[k]$ such that $\sum_{i \in e} f(i) \equiv \frac{k}{2}(\bmod k)$ for each $e \in E(H)$. We pose the following conjecture.

Conjecture. Let $H$ be a connected $k$-uniform hypergraph. Then the following are equivalent:

(1) $k$ is even and $H$ is odd-bipartite.

(2) 0 is a signless Laplacian eigenvalue of $H$.

(3) $-\rho\left(\mathcal{A}_{H}\right)$ is an eigenvalue of $H$.

(4) There exists a vertex labeling $f: V(H) \rightarrow[k]$ such that $\sum_{i \in e} f(i) \equiv \frac{k}{2}(\bmod k)$ for each $e \in E(H)$. 


\section{Eigenvalues of power hypergraphs}

A vertex with degree one is called a core vertex [7]. For a $k$-uniform hypergraph $H$, if $e \in E(H)$ contains core vertices, then we use $H-e$ to denote a $k$-uniform sub-hypergraph of $H$ obtained by deleting the edge $e$ and all core vertices in $e$.

Theorem 13. Let $H$ be a k-uniform hypergraph, and let $e \in E(H)$ be an edge contains at least two core vertices. If $\lambda$ is an eigenvalue of $H-e$, then $\lambda$ is an eigenvalue of $H$.

Proof. Suppose that $x$ is an eigenvector of the eigenvalue $\lambda$ of $H-e$. Let $y$ be a column vector of dimension $|V(H)|$ such that $y_{u}=x_{u}$ if $u \in V(H-e)$, and $y_{u}=0$ if $u \in V(H)$ is a core vertex in $e$. Since $\mathcal{A}_{H-e} x=\lambda x^{[k-1]}$, we have $\mathcal{A}_{H} y=\lambda y^{[k-1]}$. So $\lambda$ is an eigenvalue of $H$.

In [7], Hu et al defined power hypergraphs as follows.

Definition 14. [7] Let $G$ be an ordinary graph (i.e. 2-uniform hypergraph). For any $k \geqslant 3$, the $k$ th power of $G$, denoted by $G^{k}$, is a $k$-uniform hypergraph with edge set $E\left(G^{k}\right)=\left\{e \cup\left\{i_{e, 1}, \ldots, i_{e, k-2}\right\} \mid e \in E(G)\right\}$, and vertex set $V\left(G^{k}\right)=V(G) \cup\left\{i_{e, j} \mid e \in\right.$ $E(G), j \in[k-2]\}$.

Some examples of power hypergraphs are given in [7, Fig.1]. From Definition 14, we know that each edge of a power hypergraph $G^{k}$ contains two adjacent vertices in $V(G)$ and $k-2$ core vertices not in $V(G)$.

If $H$ is a connected $k$-uniform hypergraph, then $\mathcal{A}_{H}$ and $\mathcal{Q}_{H}$ are both weakly irreducible [13]. So we obtain the following lemma from [13, Theorem 2.2].

Lemma 15. Let $H$ be a connected $k$-uniform hypergraph. If $\lambda$ is an eigenvalue of $\mathcal{A}_{H}$ $\left(\mathcal{Q}_{H}\right)$ with a positive eigenvector, then $\lambda=\rho\left(\mathcal{A}_{H}\right)\left(\lambda=\rho\left(\mathcal{Q}_{H}\right)\right)$.

Theorem 16. If $\lambda \neq 0$ is an eigenvalue of a graph $G$, then $\lambda^{\frac{2}{k}}$ is an eigenvalue of $G^{k}$. Moreover, $\rho\left(\mathcal{A}_{G^{k}}\right)=\rho\left(\mathcal{A}_{G}\right)^{\frac{2}{k}}$.

Proof. Suppose that $x$ is an eigenvector of the eigenvalue $\lambda \neq 0$ of graph $G$. Then $\sum_{j \in N_{G}(i)} x_{j}=\lambda x_{i}$ for any $i \in V(G)$, where $N_{G}(i)$ is the set of all neighbors of $i$ in $G$. Let $y$ be a column vector of dimension $\left|V\left(G^{k}\right)\right|$ such that $y_{u}=\left(x_{u}\right)^{\frac{2}{k}}$ if $u \in V(G)$, and $y_{u}=\left(\lambda^{-1} x_{i} x_{j}\right)^{\frac{1}{k}}$ if $u \in V\left(G^{k}\right) \backslash V(G)$ is a core vertex in the edge contains two adjacent vertices $i, j \in V(G)$. For any $i \in V(G)$, by $\sum_{j \in N_{G}(i)} x_{j}=\lambda x_{i}$, we have

$$
\left(\mathcal{A}_{G^{k}} y\right)_{i}=\sum_{j \in N_{G}(i)}\left(\lambda^{-1} x_{i} x_{j}\right)^{\frac{k-2}{k}}\left(x_{j}\right)^{\frac{2}{k}}=\lambda^{\frac{2}{k}}\left(x_{i}\right)^{\frac{2(k-1)}{k}}=\lambda^{\frac{2}{k}}\left(y_{i}\right)^{k-1} .
$$

For any $u \in V\left(G^{k}\right) \backslash V(G)$, we have

$$
\left(\mathcal{A}_{G^{k}} y\right)_{u}=\left(\lambda^{-1} x_{i} x_{j}\right)^{\frac{k-3}{k}}\left(x_{i}\right)^{\frac{2}{k}}\left(x_{j}\right)^{\frac{2}{k}}=\lambda^{\frac{2}{k}}\left(\lambda^{-1} x_{i} x_{j}\right)^{\frac{k-1}{k}}=\lambda^{\frac{2}{k}}\left(y_{u}\right)^{k-1} .
$$

Hence $\lambda^{\frac{2}{k}}$ is an eigenvalue of $G^{k}$ with an eigenvector $y$. 
If $G$ is connected and $\lambda=\rho\left(\mathcal{A}_{G}\right)$, then we can choose $x$ as a positive eigenvector of $\rho\left(\mathcal{A}_{G}\right)$. In this case, $y$ is a positive eigenvector of the eigenvalue $\rho\left(\mathcal{A}_{G}\right)^{\frac{2}{k}}$ of $G^{k}$. Lemma 15 implies that $\rho\left(\mathcal{A}_{G^{k}}\right)=\rho\left(\mathcal{A}_{G}\right)^{\frac{2}{k}}$ when $G$ is connected.

If $G$ has $r \geqslant 2$ components $G_{1}, \ldots, G_{r}$, then

$$
\rho\left(\mathcal{A}_{G^{k}}\right)=\max \left\{\rho\left(\mathcal{A}_{G_{1}^{k}}\right), \ldots, \rho\left(\mathcal{A}_{G_{r}^{k}}\right)\right\}=\max \left\{\rho\left(\mathcal{A}_{G_{1}}\right)^{\frac{2}{k}}, \ldots, \rho\left(\mathcal{A}_{G_{r}}\right)^{\frac{2}{k}}\right\}=\rho\left(\mathcal{A}_{G}\right)^{\frac{2}{k}} .
$$

We can obtain the following result from Theorem 16 .

Corollary 17. For any nontrivial graph $G$, we have $\lim _{k \rightarrow \infty} \rho\left(\mathcal{A}_{G^{k}}\right)=1$. Moreover, $\left\{\rho\left(\mathcal{A}_{G^{k}}\right)\right\}$ is a strictly decreasing sequence if $\rho\left(\mathcal{A}_{G}\right)>1$.

The following corollary follows from Theorem 13 and 16.

Corollary 18. If $\lambda \neq 0$ is an eigenvalue of any subgraph of a graph $G$, then $\lambda^{\frac{2}{k}}$ is an eigenvalue of $G^{k}$ for $k \geqslant 4$.

Let $P_{n}$ and $S_{n}$ be the path and the star of order $n$, respectively. The following result was proved by Li et al [8]. Here we give a different proof.

Corollary 19. Let $T$ be a tree with $n$ vertices. Then

$$
\rho\left(\mathcal{A}_{P_{n}^{k}}\right) \leqslant \rho\left(\mathcal{A}_{T^{k}}\right) \leqslant \rho\left(\mathcal{A}_{S_{n}^{k}}\right),
$$

where the left equality holds if and only if $T=P_{n}$, and the right equality holds if and only if $T=S_{n}$.

Proof. Among all trees with $n$ vertices, $P_{n}$ is the unique tree with the smallest adjacency spectral radius, and $S_{n}$ is the unique tree with the largest adjacency spectral radius [2]. By Theorem 16, we have

$$
\rho\left(\mathcal{A}_{P_{n}^{k}}\right) \leqslant \rho\left(\mathcal{A}_{T^{k}}\right) \leqslant \rho\left(\mathcal{A}_{S_{n}^{k}}\right)
$$

where the left equality holds if and only if $T=P_{n}$, and the right equality holds if and only if $T=S_{n}$.

Theorem 20. If $\alpha \neq 0$ is an eigenvalue of a d-regular graph $G$, then the roots of $(x-$ $d)(x-1)^{\frac{k-2}{2}}-\alpha=0$ are signless Laplacian eigenvalues of $G^{k}$. Moreover, $\rho\left(\mathcal{Q}_{G^{k}}\right)$ is the largest real root of $(x-d)(x-1)^{\frac{k-2}{2}}-d=0$.

Proof. Suppose that $x$ is an eigenvector of the eigenvalue $\alpha \neq 0$ of graph $G$. Then $\sum_{j \in N_{G}(i)} x_{j}=\alpha x_{i}$ for any $i \in V(G)$, where $N_{G}(i)$ is the set of all neighbors of $i$ in $G$. Let $\lambda \in \mathbb{C}$ be any number such that $(\lambda-d)(\lambda-1)^{\frac{k-2}{2}}=\alpha$, then $\lambda \neq 1$. Let $y$ be a column vector of dimension $\left|V\left(G^{k}\right)\right|$ such that $y_{u}=\left(x_{u}\right)^{\frac{2}{k}}$ if $u \in V(G)$, and $y_{u}=(\lambda-1)^{-\frac{1}{2}}\left(x_{i} x_{j}\right)^{\frac{1}{k}}$ 
if $u \in V\left(G^{k}\right) \backslash V(G)$ is a core vertex in the edge contains two adjacent vertices $i, j \in V(G)$. For any $i \in V(G)$, by $\sum_{j \in N_{G}(i)} x_{j}=\alpha x_{i}$ and $(\lambda-d)(\lambda-1)^{\frac{k-2}{2}}=\alpha$, we have

$$
\left(\mathcal{Q}_{G^{k}} y\right)_{i}=d\left(x_{i}\right)^{\frac{2(k-1)}{k}}+\sum_{j \in N_{G}(i)}(\lambda-1)^{-\frac{k-2}{2}}\left(x_{i} x_{j}\right)^{\frac{k-2}{k}}\left(x_{j}\right)^{\frac{2}{k}}=\lambda\left(x_{i}\right)^{\frac{2(k-1)}{k}}=\lambda\left(y_{i}\right)^{k-1} .
$$

For any $u \in V\left(G^{k}\right) \backslash V(G)$, we have

$$
\begin{aligned}
\left(\mathcal{Q}_{G^{k}} y\right)_{u} & =(\lambda-1)^{-\frac{k-1}{2}}\left(x_{i} x_{j}\right)^{\frac{k-1}{k}}+(\lambda-1)^{-\frac{k-3}{2}}\left(x_{i} x_{j}\right)^{\frac{k-3}{k}}\left(x_{i}\right)^{\frac{2}{k}}\left(x_{j}\right)^{\frac{2}{k}} \\
& =\lambda(\lambda-1)^{-\frac{k-1}{2}}\left(x_{i} x_{j}\right)^{\frac{k-1}{k}}=\lambda\left(y_{u}\right)^{k-1} .
\end{aligned}
$$

Hence $\lambda$ is a signless Laplacian eigenvalue of $G^{k}$ with an eigenvector $y$.

If $G$ is connected and $\alpha=d=\rho\left(\mathcal{A}_{G}\right)$, then we can choose $x$ as a positive eigenvector of $\rho\left(\mathcal{A}_{G}\right)$. In this case, $y$ is a positive eigenvector of the signless Laplacian eigenvalue $\lambda$ of $G^{k}$. Lemma 15 implies that $\rho\left(\mathcal{Q}_{G^{k}}\right)$ is the largest real root of $(x-d)(x-1)^{\frac{k-2}{2}}-d=0$ when $G$ is connected.

If $G$ has $r \geqslant 2$ components $G_{1}, \ldots, G_{r}$, then

$$
\rho\left(\mathcal{Q}_{G^{k}}\right)=\max \left\{\rho\left(\mathcal{Q}_{G_{1}^{k}}\right), \ldots, \rho\left(\mathcal{Q}_{G_{r}^{k}}\right)\right\} .
$$

Since $G_{1}, \ldots, G_{r}$ are connected $d$-regular graphs, we know that $\rho\left(\mathcal{Q}_{G^{k}}\right)=\rho\left(\mathcal{Q}_{G_{1}^{k}}\right)=\cdots=$ $\rho\left(\mathcal{Q}_{G_{r}^{k}}\right)$ is equal to the largest real root of $(x-d)(x-1)^{\frac{k-2}{2}}-d=0$.

The following corollary follows from Theorem 20 .

Corollary 21. For any d-regular graph $G$, we have $\lim _{k \rightarrow \infty} \rho\left(\mathcal{Q}_{G^{k}}\right)=d$. Moreover, $\rho\left(\mathcal{Q}_{G^{k}}\right)$ is a strictly decreasing sequence if $d>1$.

Remark. In [7, Conjecture 4.1], Hu et al conjectured that $\rho\left(\mathcal{Q}_{G^{k}}\right)$ is a strictly decreasing sequence for any graph $G$ and even $k$. By Corollary 21, this conjecture holds when $G$ is regular of degree $d>1$.

The proof of the following theorem is similar with that of Theorem 20. So we omit it.

Theorem 22. If $\alpha \neq 0$ is an eigenvalue of a d-regular graph $G$, then the roots of $(d-$ $x)(1-x)^{\frac{k-2}{2}}-\alpha=0$ are Laplacian eigenvalues of $G^{k}$.

\section{Acknowledgements}

This work is supported by the National Natural Science Foundation of China (No. 11371109), the Natural Science Foundation of the Heilongjiang Province (No. QC2014C001), and the Fundamental Research Funds for the Central Universities (No. 2014110015). 


\section{References}

[1] J. Cooper and A. Dutle. Spectra of uniform hypergraphs. Linear Algebra Appl., 436:3268-3292, 2012.

[2] D. Cvetković, P. Rowlinson, and S. Simić. An Introduction to the Theory of Graph Spectra. Cambridge University Press, Cambridge, 2010.

[3] S. Friedland, S. Gaubert, and L. Han. Perron-Frobenius theorem for nonnegative multilinear forms and extensions. Linear Algebra Appl., 438:738-749, 2013.

[4] S. Hu, Z. Huang, C. Ling, and L. Qi. On determinants and eigenvalue theory of tensors. J. Symbolic Comput., 50:508-531, 2013.

[5] S. Hu and L. Qi. The Laplacian of a uniform hypergraph. J. Comb. Optim., 2013. doi:10.1007/s10878-013-9596-x.

[6] S. Hu and L. Qi. The eigenvectors associated with the zero eigenvalues of the Laplacian and signless Laplacian tensors of a uniform hypergraph. Discrete Appl. Math., 169:140-151, 2014.

[7] S. Hu, L. Qi, and J.Y. Shao. Cored hypergraphs, power hypergraphs and their Laplacian H-eigenvalues. Linear Algebra Appl., 439:2980-2998, 2013.

[8] H. Li, J.Y. Shao, and L. Qi. The extremal spectral radii of $k$-uniform supertrees. arXiv: 1405.7257.

[9] L.H. Lim. Singular values and eigenvalues of tensors: a variational approach. In Proceedings of the IEEE International Workshop on Computational Advances in Multisensor Adaptive Processing, December 13-15, pages 129-132, 2005.

[10] A. Morozov and Sh. Shakirov. Analogue of the identity Log Det=Trace Log for resultants. J. Geom. Phys., 61:708-726, 2011.

[11] K. Pearson and T. Zhang. On spectral hypergraph theory of the adjacency tensor. Graphs Combin., 30:1233-1248, 2014.

[12] L. Qi. Eigenvalues of a real supersymmetric tensor. J. Symbolic Comput., 40:13021324, 2005.

[13] L. Qi. $\mathrm{H}^{+}$-eigenvalues of Laplacian and signless Laplacian tensors. Commun. Math. Sci., 12:1045-1064, 2014.

[14] L. Qi, J.Y. Shao, and Q. Wang. Regular uniform hypergraphs, s-cycles, s-paths and their largest Laplacian H-eigenvalues. Linear Algebra Appl., 443:215-227, 2014.

[15] J.Y. Shao. A general product of tensors with applications. Linear Algebra Appl., 439:2350-2366, 2013.

[16] J.Y. Shao, L. Qi, and S. Hu. Some new trace formulas of tensors with applications in spectral hypergraph theory. Linear and Multilinear Algebra, 2014. doi:10.1080/03081087.2014.910208.

[17] J.Y. Shao, H.Y. Shan, and B. Wu. Some spectral properties and characterizations of connected odd-bipartite uniform hypergraphs. arXiv:1403.4845. 
[18] J. Xie and A. Chang. On the Z-eigenvalues of the adjacency tensors for uniform hypergraphs. Linear Algebra Appl., 439:2195-2204, 2013.

[19] Y. Yang and Q. Yang. On some properties of nonnegative weakly irreducible tensors. arXiv: 1111.0713v2. 